\title{
The Ising spin glass in finite dimensions: a perturbative study of the free energy
}

\author{
T. Temesvári* \\ Research Group for Theoretical Physics of the Hungarian Academy of Sciences, \\ Eötvös University, Pázmány Péter sétány 1/A, H-1117 Budapest, Hungary
}

(Dated: November 15, 2018)

\begin{abstract}
Replica field theory is used to study the $n$-dependent free energy of the Ising spin glass in a first order perturbative treatment. Large sample-to-sample deviations of the free energy from its quenched average prove to be Gaussian, independently of the special structure of the order parameter. The free energy difference between the replica symmetric and (infinite level) replica symmetry broken phases is studied in details: the line $n(T)$ where it is zero coincides with the Almeida-Thouless line for $d>8$. The dimensional domain $6<d<8$ is more complicated, and several scenarios are possible.
\end{abstract}

PACS numbers: $75.10 . \mathrm{Nr}$

\section{INTRODUCTION}

Large scale free energy deviations $F \equiv N f$ of a quenched disordered system from its selfaveraged mean $N f_{\text {typ }}$ are extremely rare events with probability $\sim e^{N L(f)}$, with the system size $N \rightarrow \infty$, and $L(f)=O(1)<0$ for $f=O(1) \neq f_{\text {typ }}$. Only the case $f<f_{\text {typ }}$ is considered in this paper. As invented in Ref. [1], $L(f)$ can be computed by the replica method; namely it is the Legendre transform of the $n$-dependent free energy $\Phi$ of (1):

$$
\beta^{-1} L(f)=-n \Phi+n f \quad \text { and } \quad \frac{d}{d n}(n \Phi)=f .
$$

The replica number $n$ is considered a nonnegative real number corresponding to $f \leq f_{\text {typ }}$, see also [2, 3]. In most applications, we have $\Phi-f_{\text {typ }} \sim-n^{a}$ for $n \rightarrow 0^{+}$with some positive integer exponent $a$ characteristic to the model, and also to the region of control parameters (temperature, magnetic field, etc.) considered. Legendre transforming then provides $L(f) \sim-\left(f_{\text {typ }}-f\right)^{\frac{a+1}{a}}$. In the most common situations [1, 2, 3], $a=1$ and the large scale free energy fluctuations are Gaussian.

A very atypical behaviour was found many years ago by Kondor [4] for the truncated version of the Sherrington-Kirkpatrick (SK) model [5], i.e. for the mean field Ising spin glass, just below the transition

${ }^{*}$ Electronic address: temtam@helios.elte.hu 
temperature. The exponent $a=5$ was later proved to be true in the whole spin glass phase, including zero temperature [2, 3]. The original motivation of the present work was to find out how the large free energy deviations are influenced by including the geometry of a $d$-dimensional hypercubic lattice. For that purpose, we followed the usual steps to generate a field theory convinient for a perturbative calculation whose zeroth order is the mean field (SK) theory. These steps are:

- Transformation of the lattice system to a field theory, see Refs. [6, 7]. The natural ultraviolet cutoff is then the boundary of the Brillouin zone, and it is $\Lambda=O(1)$ when length is measured in the units of the lattice spacing.

- The momentum space is shrinking such that $T_{c}-T \sim \tau \ll \Lambda \ll 1$.

- Momentum dependence is truncated up to the quadratic term.

We are led then to the replicated field theory presented in the next section, Eqs. (2) and (3). (Zero external magnetic field is assumed throughout the present paper.) It turns out from the calculation of Sec. IV] that the leading behaviour of the large scale fluctuations changes to Gaussian when first order corrections to the low temperature SK model are taken into account (this has been noticed in Ref. [8]), and the anomalous $n^{5}$ term is now subleading. It becomes also clear that this Gaussian behaviour follows for any structure of the order parameter as long as replica equivalence is assumed.

The glassy phase of the SK model has the ultrametric structure with infinite step replica symmetry breaking [9] $(\mathrm{RSB})^{1}$. For $T<T_{c}$, this RSB state is reached by a phase transition [4] when $n$ is lowered from around 1 - where $\Phi(n)$ is the free energy of the stable replica symmetric (RS) phase with a nonzero order parameter - at some $n_{\mathrm{AT}}(T)$ having two characteristics:

1. The RS phase becomes unstable due to the Almeida-Thouless [10] instability, i.e. the so called replicon mass $\Gamma_{\mathrm{R}}$ is zero for $n=n_{\mathrm{AT}}(T)$.

2. $\Phi_{\mathrm{RS}}=\Phi_{\mathrm{RSB}}$ at the transition.

Whether or not this feature of the SK model is perturbatively stable is studied in the second part of the paper, and rather nontrivial calculations lead us to a positive conclusion for $d>8$. The transitional domain of dimensions above the upper critical dimension $(6<d<8)$ is, however, more complicated: it is argued that the question cannot be settled by this first order calculation.

\footnotetext{
${ }^{1}$ The acronym RSB is used throughout the paper for the special type of replica symmetry breaking with infinite number of levels in the ultrametric hierarchy.
} 
The outline of the paper is as follows: Sec. II presents the replicated field theoretical model suitable for the perturbative calculation, whose basic formulae are provided in Sec. III. The one-loop results for the $n$-dependent free energy are shown in Sec. IV] up to fourth order in the double series of $\tau \sim T_{c}-T$ and $n$ : these are the leading contributions when $d>8$. Nonanalytic temperature dependences are more and more important while approaching dimension 8 , and even for $6<d<8$. They are computed for both the RSB and RS schemes in Sec. $\mathrm{V}$. The line of equal free energies of the two schemes is calculated for $d>8$ in Sec. VI, whereas we analyze the situation in the transitional regime just above the upper critical dimension, i.e. $6<d<8$, in Sec. VII. One-loop results for the RS replicon mass $\Gamma_{\mathrm{R}}$ are presented and the AT instability line deduced in Sec. VIII. Some concluding remarks are left to Sec. IX. Three appendices contain some computational details.

\section{FIELD THEORETIC MODEL FOR THE CALCULATION OF THE FREE ENERGY}

In this section, we will define the replicated field theory which is appropriate for the calculation of perturbative corrections to the $n$-dependent free energy $\Phi(n, \beta)$ defined as

$$
\beta \Phi(n, \beta) \equiv-\frac{1}{n N} \ln \overline{Z^{n}}
$$

where $Z$ is the partition function of an Ising spin glass defined on the $d$-dimensional hypercubic lattice consisting of $N$ Ising spins, and the bar denotes averaging over the quenched Gaussian disorder with zero mean and variance $J^{2}$. The thermodynamic limit $N \rightarrow \infty$ is always understood, whereas $n>0$ is small but kept finite. We follow the strategy suggested in Ref. [11]: by conviniently choosing the bare parameters, the field theory provides the SK results [2, 3, 4] in the tree approximation, i.e. when neglecting loops. In zero external magnetic field we can express $\overline{Z^{n}}$ in the following functional integral form (see Refs. [7, 12]):

$$
\overline{Z^{n}}=C \times \int[d \phi] e^{-\mathcal{L}}
$$

with the normalization factor $C$ to be fixed later by choosing it to match the SK result. The Lagrangian $\mathcal{L}$ has the form

$$
\begin{aligned}
& \mathcal{L}=\frac{1}{2} \sum_{\mathbf{p}}\left(\frac{1}{2} p^{2}+m\right) \sum_{\alpha \beta} \phi_{\mathbf{p}}^{\alpha \beta} \phi_{-\mathbf{p}}^{\alpha \beta}-\frac{1}{6 \sqrt{N}} \sum_{\mathbf{p}_{1} \mathbf{p}_{2} \mathbf{p}_{3}}^{\prime} w \sum_{\alpha \beta \gamma} \phi_{\mathbf{p}_{1}}^{\alpha \beta} \phi_{\mathbf{p}_{2}}^{\beta \gamma} \phi_{\mathbf{p}_{3}}^{\gamma \alpha}-\frac{1}{24 N} \sum_{\mathbf{p}_{1} \mathbf{p}_{2} \mathbf{p}_{3} \mathbf{p}_{4}}^{\prime} \\
& \left(u_{1} \sum_{\alpha \beta \gamma \delta} \phi_{\mathbf{p}_{1}}^{\alpha \beta} \phi_{\mathbf{p}_{2}}^{\beta \gamma} \phi_{\mathbf{p}_{3}}^{\gamma \delta} \phi_{\mathbf{p}_{4}}^{\delta \alpha}+u_{2} \sum_{\alpha \beta} \phi_{\mathbf{p}_{1}}^{\alpha \beta} \phi_{\mathbf{p}_{2}}^{\alpha \beta} \phi_{\mathbf{p}_{3}}^{\alpha \beta} \phi_{\mathbf{p}_{4}}^{\alpha \beta}+u_{3} \sum_{\alpha \beta \gamma} \phi_{\mathbf{p}_{1}}^{\alpha \gamma} \phi_{\mathbf{p}_{2}}^{\alpha \gamma} \phi_{\mathbf{p}_{3}}^{\beta \gamma} \phi_{\mathbf{p}_{4}}^{\beta \gamma}+u_{4} \sum_{\alpha \beta \gamma \delta} \phi_{\mathbf{p}_{1}}^{\alpha \beta} \phi_{\mathbf{p}_{2}}^{\alpha \beta} \phi_{\mathbf{p}_{3}}^{\gamma \delta} \phi_{\mathbf{p}_{4}}^{\gamma \delta}\right)+\ldots
\end{aligned}
$$


where the ellipses are for higher order replica symmetric invariants which are consistent with the extra symmetry of the zero magnetic field subspace [12, 13]. In this $n(n-1) / 2$ component field theory the fluctuating fields are symmetric in the replica indices with zero diagonals: $\phi_{\mathbf{p}}^{\alpha \beta}=\phi_{\mathbf{p}}^{\beta \alpha}$ and $\phi_{\mathbf{p}}^{\alpha \alpha}=0$, $\alpha, \beta=1, \ldots, n$. (Momentum conservation is indicated by the primed summations.) The bare mass $m$ depends on temperature as $m=-\frac{k^{2}}{2 J^{2}}\left(T_{c}^{\mathrm{mf}^{2}}-T^{2}\right)$ where $k$ is Boltzmann's constant, and $k T_{c}^{\mathrm{mf}}=J-$ the mean field critical temperature - differs from the exact one, $T_{c}$. Introducing the new parameter $\tau \equiv \frac{k^{2}}{2 J^{2}}\left(T_{c}^{2}-T^{2}\right)$ measuring the distance from criticality, the bare mass can be written as $m=m_{c}-\tau$ with $m_{c}$ being obviously one-loop order. Beside $\tau$, the couplings $w, u_{1}, u_{2}, u_{3}, u_{4}, \ldots$ parametrize the Lagrangian. Around the critical point they can be considered as constants; a complete matching with the SK results is achieved by choosing $w=1, u_{1}=3, u_{2}=2, u_{3}=-6, u_{4}=0$ [11].

The spin glass phase below $T_{c}$ is characterized by the nonzero order parameter $\phi^{\alpha \beta} \equiv \frac{1}{\sqrt{N}}\left\langle\phi_{\mathbf{p}=0}^{\alpha \beta}\right\rangle$ where the average is now taken by the measure proportional to $e^{-\mathcal{L}}$. It is useful to redefine the fields by the shift $\phi_{\mathbf{p}}^{\alpha \beta} \longrightarrow \phi_{\mathbf{p}}^{\alpha \beta}-\sqrt{N} \phi^{\alpha \beta} \delta_{\mathbf{p}=0}^{\mathrm{Kr}}$. By this transformation, the new fields continue fluctuating around zero; on the other hand, however, the Lagrangian has lost the higher symmetry of the paramagnetic phase resulting in the following generic theory (equally convinient for an RS or RSB ansatz): ${ }^{2}$

$$
\mathcal{L}=\mathcal{L}^{(0)}+\mathcal{L}^{(1)}+\mathcal{L}^{(2)}+\mathcal{L}^{(3)}+\mathcal{L}^{(4)}+\ldots
$$

\footnotetext{
${ }^{2}$ For the sake of simplifying the notations, we will keep the symbols $\phi_{\mathbf{p}}^{\alpha \beta}$ and $\mathcal{L}$ for the transformed quantities.
} 
The terms above can be worked out using the results of the Appendices B and D of Ref. [13] providing

$$
\begin{aligned}
& \frac{1}{N} \mathcal{L}^{(0)}=\frac{1}{2} m \sum_{\alpha \beta} \phi^{\alpha \beta^{2}}-\frac{1}{6} w \sum_{\alpha \beta \gamma} \phi^{\alpha \beta} \phi^{\beta \gamma} \phi^{\gamma \alpha}-\frac{1}{24}\left(u_{1} \sum_{\alpha \beta \gamma \delta} \phi^{\alpha \beta} \phi^{\beta \gamma} \phi^{\gamma \delta} \phi^{\delta \alpha}+u_{2} \sum_{\alpha \beta} \phi^{\alpha \beta^{4}}\right. \\
& \left.+u_{3} \sum_{\alpha \beta \gamma} \phi^{\alpha \gamma 2} \phi^{\beta \gamma^{2}}+u_{4} \sum_{\alpha \beta \gamma \delta} \phi^{\alpha \beta^{2}} \phi^{\gamma \delta^{2}}\right)+\ldots, \\
& \frac{1}{\sqrt{N}} \mathcal{L}^{(1)}=\sum_{\alpha \beta}\left[m \phi^{\alpha \beta}-\frac{1}{2} w \sum_{\gamma} \phi^{\alpha \gamma} \phi^{\gamma \beta}-\frac{1}{6}\left(u_{1} \sum_{\gamma \delta} \phi^{\alpha \gamma} \phi^{\gamma \delta} \phi^{\delta \beta}+u_{2} \phi^{\alpha \beta^{3}}+u_{3} \phi^{\alpha \beta} \sum_{\gamma} \phi^{\beta \gamma^{2}}\right.\right. \\
& \left.\left.+u_{4} \phi^{\alpha \beta} \sum_{\gamma \delta} \phi^{\gamma \delta^{2}}\right)+\ldots\right] \times \phi_{\mathbf{p}=0}^{\alpha \beta}, \\
& \mathcal{L}^{(2)}=\frac{1}{2} \sum_{\mathbf{p}}\left[\sum_{\alpha \beta}\left(\frac{1}{2} p^{2}+m-\frac{1}{2} u_{2} \phi^{\alpha \beta^{2}}-\frac{1}{6} u_{3} \sum_{\gamma} \phi^{\beta \gamma^{2}}-\frac{1}{6} u_{4} \sum_{\gamma \delta} \phi^{\gamma \delta^{2}}+\ldots\right) \times \phi_{\mathbf{p}}^{\alpha \beta} \phi_{-\mathbf{p}}^{\alpha \beta}\right. \\
& +\sum_{\alpha \beta \gamma}\left(-w \phi^{\alpha \beta}-\frac{1}{3} u_{1} \sum_{\delta} \phi^{\alpha \delta} \phi^{\delta \beta}-\frac{1}{3} u_{3} \phi^{\alpha \gamma} \phi^{\gamma \beta}+\ldots\right) \times \phi_{\mathbf{p}}^{\alpha \gamma} \phi_{-\mathbf{p}}^{\gamma \beta} \\
& \left.+\sum_{\alpha \beta \gamma \delta}\left(-\frac{1}{6} u_{1} \phi^{\alpha \gamma} \phi^{\beta \delta}-\frac{1}{3} u_{4} \phi^{\alpha \beta} \phi^{\gamma \delta}+\ldots\right) \times \phi_{\mathbf{p}}^{\alpha \beta} \phi_{-\mathbf{p}}^{\gamma \delta}\right], \\
& \sqrt{N} \mathcal{L}^{(3)}=-\frac{1}{6} \sum_{\mathbf{p}_{1} \mathbf{p}_{2} \mathbf{p}_{3}}^{\prime}\left[\sum_{\alpha \beta \gamma}(w+\ldots) \times \phi_{\mathbf{p}_{1}}^{\alpha \beta} \phi_{\mathbf{p}_{2}}^{\beta \gamma} \phi_{\mathbf{p}_{3}}^{\gamma \alpha}+\sum_{\alpha \beta}\left(u_{2} \phi^{\alpha \beta}+\ldots\right) \times \phi_{\mathbf{p}_{1}}^{\alpha \beta} \phi_{\mathbf{p}_{2}}^{\alpha \beta} \phi_{\mathbf{p}_{3}}^{\alpha \beta}\right. \\
& +\sum_{\alpha \beta \gamma}\left(u_{3} \phi^{\beta \gamma}+\ldots\right) \times \phi_{\mathbf{p}_{1}}^{\alpha \beta} \phi_{\mathbf{p}_{2}}^{\alpha \beta} \phi_{\mathbf{p}_{3}}^{\beta \gamma}+\sum_{\alpha \beta \gamma \delta}\left(u_{4} \phi^{\gamma \delta}+\ldots\right) \times \phi_{\mathbf{p}_{1}}^{\alpha \beta} \phi_{\mathbf{p}_{2}}^{\alpha \beta} \phi_{\mathbf{p}_{3}}^{\gamma \delta} \\
& \left.+\sum_{\alpha \beta \gamma \delta}\left(u_{1} \phi^{\gamma \delta}+\ldots\right) \times \phi_{\mathbf{p}_{1}}^{\alpha \beta} \phi_{\mathbf{p}_{2}}^{\alpha \gamma} \phi_{\mathbf{p}_{3}}^{\beta \delta}+\ldots\right] .
\end{aligned}
$$

$\mathcal{L}^{(4)}$ has been omitted here, as it keeps its form in Eq. (3) up to this order, whereas the ellipsis dots indicate the terms higher than quartic.

\section{PERTURBATIVE CALCULATION OF THE FREE ENERGY}

Two steps lead to a systematic perturbative treatment:

- Firstly an interaction Lagrangian is detached, and $\mathcal{L}^{(2)}$ identified as the non-interractive part:

$$
\mathcal{L}=\mathcal{L}^{(0)}+\frac{1}{2} \sum_{\mathbf{p}} \sum_{(\alpha \beta),(\gamma \delta)}\left(p^{2} \delta_{\alpha \beta, \gamma \delta}^{\mathrm{Kr}}+\bar{M}_{\alpha \beta, \gamma \delta}\right) \phi_{\mathbf{p}}^{\alpha \beta} \phi_{-\mathbf{p}}^{\gamma \delta}+\mathcal{L}^{\mathrm{I}}
$$

where in the second term the bare mass is now represented by the mass operator $\bar{M}_{\alpha \beta, \gamma \delta}$, and the summation $\sum_{(\alpha \beta)} \equiv \sum_{\alpha<\beta}$ is over the $n(n-1) / 2$ pairs of replica indices, i.e., over the independent field components. Comparing with Eq. (66), the three different types of mass components are simply 
derived:

$$
\begin{aligned}
& \bar{M}_{\alpha \beta, \alpha \beta}=2 m-\frac{1}{3}\left(u_{1}+3 u_{2}+2 u_{3}+4 u_{4}\right) \phi^{\alpha \beta^{2}}-\frac{1}{3}\left(2 u_{1}+u_{3}+n u_{4}\right) \sum_{\gamma} \phi^{\beta \gamma^{2}}+\ldots, \\
& \bar{M}_{\alpha \gamma, \beta \gamma}=-w \phi^{\alpha \beta}-\frac{1}{3}\left(u_{1}+u_{3}+4 u_{4}\right) \phi^{\alpha \gamma} \phi^{\beta \gamma}-\frac{1}{3} u_{1} \sum_{\delta} \phi^{\alpha \delta} \phi^{\beta \delta}+\ldots \\
& \bar{M}_{\alpha \beta, \gamma \delta}=-\frac{1}{3} u_{1}\left(\phi^{\alpha \gamma} \phi^{\beta \delta}+\phi^{\beta \gamma} \phi^{\alpha \delta}\right)-\frac{4}{3} u_{4} \phi^{\alpha \beta} \phi^{\gamma \delta}+\ldots
\end{aligned}
$$

Replica equivalence is a must even when replica symmetry is broken [14], and it has been assumed in the above formulas; see the last term of the diagonal component which is in fact independent of $\beta$. The free propagator of this generic RSB field theory is the $n(n-1) / 2 \times n(n-1) / 2$ matrix $\bar{G} \equiv\left(p^{2}+\bar{M}\right)^{-1}$.

- The order parameter $\phi^{\alpha \beta}$ must satisfy the "equation of state" $\left\langle\phi_{\mathbf{p}=0}^{\alpha \beta}\right\rangle=0$; up to one-loop order it takes the form

$$
\bar{H}_{\alpha \beta}+\frac{1}{2} \sum_{(\gamma \delta),(\mu \nu)} \bar{W}_{\alpha \beta, \gamma \delta, \mu \nu} \times \frac{1}{N} \sum_{\mathbf{p}} \bar{G}_{\gamma \delta, \mu \nu}(p)+2 \text {-loop terms }=0 .
$$

In perturbative computations, the capital letter representation of the couplings is more natural, the one- and three-point ones can be deduced by comparing with Eqs. (5) and (7):

$$
\begin{aligned}
-\bar{H}_{\alpha \beta}= & 2 \times\left[m \phi^{\alpha \beta}-\frac{1}{2} w \sum_{\gamma} \phi^{\alpha \gamma} \phi^{\gamma \beta}-\frac{1}{6}\left(u_{1} \sum_{\gamma \delta} \phi^{\alpha \gamma} \phi^{\gamma \delta} \phi^{\delta \beta}+u_{2} \phi^{\alpha \beta^{3}}+u_{3} \phi^{\alpha \beta} \sum_{\gamma} \phi^{\beta \gamma^{2}}\right.\right. \\
& \left.\left.+u_{4} \phi^{\alpha \beta} \sum_{\gamma \delta} \phi^{\gamma \delta^{2}}\right)+\ldots\right]
\end{aligned}
$$

and the eight different types of cubic couplings:

$$
\begin{array}{llrl}
\bar{W}_{\alpha \beta, \beta \gamma, \gamma \alpha} & =w+\ldots, & \bar{W}_{\alpha \beta, \alpha \beta, \alpha \beta} & =2\left(u_{1}+u_{2}+u_{3}+2 u_{4}\right) \phi^{\alpha \beta}+\ldots, \\
\bar{W}_{\alpha \beta, \alpha \beta, \beta \gamma} & =\frac{1}{3}\left(2 u_{1}+u_{3}+4 u_{4}\right) \phi^{\beta \gamma}+\ldots, & \bar{W}_{\alpha \beta, \alpha \beta, \gamma \delta}=\frac{4}{3} u_{4} \phi^{\gamma \delta}+\ldots, \\
\bar{W}_{\alpha \beta, \alpha \gamma, \beta \delta}=\frac{1}{3} u_{1} \phi^{\gamma \delta}+\ldots & &
\end{array}
$$

where the dots are for the next $-O\left(\phi^{2}\right)$ - order, and the three missing cubic vertices $-\bar{W}_{\alpha \beta, \alpha \gamma, \alpha \delta}$, $\bar{W}_{\alpha \gamma, \beta \gamma, \mu \nu}$, and $W_{\alpha \beta, \gamma \delta, \mu \nu}$ - enter only at this higher order.

The critical temperature of the field theory, or equivalently $m_{c}$, can be simply deduced from Eq. (10) by expanding it up to its leading $O(\phi)$ order after setting $\tau=0$. Making use of the large momentum expansion of the free propagator $\bar{G}$, together with Eqs. (9) and (12), it straightforwardly follows:

$$
m_{c}=\frac{1}{2}(n-2) w^{2} \frac{1}{N} \sum_{\mathbf{p}} \frac{1}{p^{4}}+\frac{1}{6}\left[(2 n-1) u_{1}+3 u_{2}+(n+1) u_{3}+\left(n^{2}-n+4\right) u_{4}\right] \frac{1}{N} \sum_{\mathbf{p}} \frac{1}{p^{2}}+2 \text {-loop terms. }
$$


The free energy $\ln \overline{Z^{n}}$ has the following expansion when $\mathcal{L}^{\mathrm{I}}$ is handled as a perturbation; see (2) and (8)):

$$
\ln \overline{Z^{n}}=\ln C-\mathcal{L}^{(0)}+\ln Z_{G}-\left\langle L^{\mathrm{I}}\right\rangle_{G}+\frac{1}{2}\left(\left\langle L^{\mathrm{I}^{2}}\right\rangle_{G}-\left\langle L^{\mathrm{I}}\right\rangle_{G}^{2}\right)+\ldots,
$$

and the Gaussian averages $\langle\ldots\rangle_{G}$ are taken by the measure $e^{-\mathcal{L}^{(2)}} / Z_{G}$. As "tadpole" diagrams are missing now due to Eq. (10), the only one-loop term is the Gaussian free energy $\ln Z_{G}=\int[d \phi] e^{-\mathcal{L}^{(2)}}$ which has the familiar form in terms of the eigenvalues $\bar{\lambda}_{j}$ 's of the mass operator $\bar{M}$ :

$$
\ln Z_{G}=-\frac{1}{2} \sum_{\mathbf{p}} \sum_{j=1}^{n(n-1) / 2} \ln \frac{p^{2}+\bar{\lambda}_{j}}{\pi} .
$$

Using the following identities:

$$
\begin{aligned}
\sum_{\mathbf{p}} \ln \left(1+\frac{\bar{\lambda}_{j}}{p^{2}}\right) & =\ln \left(1+\frac{\bar{\lambda}_{j}}{\Lambda^{2}}\right) \times\left(\sum_{\mathbf{p}} 1\right)+\bar{\lambda}_{j} \frac{2}{d} \sum_{\mathbf{p}} \frac{1}{p^{2}+\bar{\lambda}_{j}}, \\
\frac{1}{p^{2}+\bar{\lambda}_{j}} & =\frac{1}{p^{2}}-\frac{\bar{\lambda}_{j}}{p^{4}}+\frac{\bar{\lambda}_{j}^{2}}{p^{6}}-\frac{\bar{\lambda}_{j}^{3}}{p^{6}\left(p^{2}+\bar{\lambda}_{j}\right)}
\end{aligned}
$$

where $\Lambda$ is the ultraviolet cutoff, and frequently applying the trivial relationship $\sum_{\mathbf{p}} 1=\frac{d-k}{d} \Lambda^{k} \sum_{\mathbf{p}} \frac{1}{p^{k}}$ with $d>k$, the Gaussian free energy can be arranged into its final form for $d>8$

$$
\begin{aligned}
\ln Z_{G} & =\frac{n(n-1)}{2}\left(\frac{1}{d}-\frac{1}{2} \ln \frac{\Lambda^{2}}{\pi}\right) \times \sum_{\mathbf{p}} 1-\frac{1}{2}\left(\sum_{j} \bar{\lambda}_{j}\right) \times \sum_{\mathbf{p}} \frac{1}{p^{2}}+\frac{1}{4}\left(\sum_{j} \bar{\lambda}_{j}^{2}\right) \times \sum_{\mathbf{p}} \frac{1}{p^{4}} \\
& -\frac{1}{6}\left(\sum_{j} \bar{\lambda}_{j}^{3}\right) \times \sum_{\mathbf{p}} \frac{1}{p^{6}}+\frac{1}{8}\left(\sum_{j} \bar{\lambda}_{j}^{4}\right) \frac{d-8}{d} \times \sum_{\mathbf{p}} \frac{1}{p^{8}}+\frac{1}{d} \sum_{j} \sum_{\mathbf{p}} \frac{\bar{\lambda}_{j}^{4}}{p^{6}\left(p^{2}+\bar{\lambda}_{j}\right)}+O\left(\bar{\lambda}_{j}^{5}\right) \times \sum_{\mathbf{p}} 1 .
\end{aligned}
$$

Before displaying the result for the free energy, it must be noticed that the contribution $N n \frac{1}{2} m_{c}\left(\phi^{2}\right)^{\alpha \alpha}$ from $\mathcal{L}^{(0) 3}$ is exactly cancelled by corresponding terms of $\ln Z_{G}$; see Eqs. (41), (13) and the second terms of the right hand sides of (A1), (A2). Moreover, as $\mathcal{L}^{(0)}$ is stationary at the zero-loop order parameter, we do not need to compute the one-loop correction to $\phi^{\alpha \beta}$. Substituting the traces from App. A into Eq.

\footnotetext{
${ }^{3}$ Matrix notations, like $\left(\phi^{2}\right)^{\alpha \alpha}$ here, are frequently used in the following part of the paper.
} 
(16), the replicated free energy in (14) takes the following form:

$$
\begin{aligned}
& \frac{1}{n N} \ln \overline{Z^{n}}=\frac{1}{n N}\left[\ln \overline{Z^{n}}\right]^{\text {para }} \\
& +\frac{1}{2} \tau \times\left(\phi^{2}\right)^{\alpha \alpha}+\frac{1}{6} w\left\{1-\frac{1}{2}\left[u_{1}+3 u_{2}+(n-1) u_{3}+\left(n^{2}-n-4\right) u_{4}\right] I_{4}-2(n-2) w^{2} I_{6}\right\} \times\left(\phi^{3}\right)^{\alpha \alpha} \\
& +\frac{1}{24}\left\{u_{1}-\frac{1}{3} u_{1}\left[(2 n-3) u_{1}+6 u_{2}+2(n-1) u_{3}+2\left(n^{2}-n-8\right) u_{4}\right] I_{4}-2 w^{2}\left[(2 n-7) u_{1}-3 u_{2}-(n-1) u_{3}\right.\right. \\
& \left.\left.-\left(n^{2}-n+4\right) u_{4}\right] I_{6}+3(3 n-4) w^{4} I_{8}\right\} \times\left(\phi^{4}\right)^{\alpha \alpha}+\frac{1}{24}\left\{u_{2}+\frac{1}{3}\left[2 u_{1}^{2}-4(n-2) u_{1} u_{2}+3 u_{2}^{2}-2(n-5) u_{2} u_{3}\right.\right. \\
& \left.\left.\quad-2\left(n^{2}-n-8\right) u_{2} u_{4}+2 u_{3}^{2}\right] I_{4}-4 w^{2}\left[-2 u_{1}+(n+1) u_{2}+2 u_{3}\right] I_{6}+24 w^{4} I_{8}\right\} \times \sum_{\beta} \phi^{\alpha \beta^{4}} \\
& +\frac{1}{24}\left\{\left(u_{3}+n u_{4}\right)+\frac{1}{3}\left[(3 n-4) u_{1}^{2}+12 u_{1} u_{2}+8 u_{1} u_{3}-(n-3) u_{3}^{2}-2\left(n^{2}-n-8\right) u_{3} u_{4}-n\left(n^{2}-n-8\right) u_{4}^{2}\right] I_{4}\right. \\
& \left.\quad-2 w^{2}\left[-(6 n-19) u_{1}-3 u_{2}+(n-5) u_{3}+\left(n^{2}-n+4\right) u_{4}\right] I_{6}+3(n-12) w^{4} I_{8}\right\} \times\left(\left(\phi^{2}\right)^{\alpha \alpha}\right)^{2} . \quad(17)
\end{aligned}
$$

Some remarks are appropriate here:

- Only replica equivalence was used to derive the above formula which gives the first order - i.e., one-loop - correction to the mean field free energy. Any special RSB scheme is included in the "invariants" like $\left(\phi^{3}\right)^{\alpha \alpha}$.

- For $\phi \equiv 0$, the analytic continuation of the paramagnetic free energy is obtained:

$$
\left[\ln \overline{Z^{n}}\right]^{\text {para }}=\ln C-\frac{n(n-1)}{4} \sum_{\mathbf{p}}^{\Lambda} \ln \frac{p^{2}-2 \tau}{\pi} ;
$$

an expansion of this formula gives the $\tau$ terms in $\ln Z_{G}$, see (16) and App. A. $n=1$ is the annealed model; the disappearance of the loop corrections expresses the triviality of this case: $\ln \bar{Z}=$ $N \ln 2+\frac{N_{B}}{z} \frac{J^{2}}{2(k T)^{2}}$, where the number of interactions $N_{B}$ can be expressed by the coordination number $z$ as $N_{B}=N \frac{z}{2}$. This gives for $\ln C$ :

$$
\frac{1}{n N} \ln C=\ln 2+\frac{J^{2}}{4(k T)^{2}}
$$

which agrees with the correct value of the SK model for $n$ generic.

- The notation $I_{k}=\frac{1}{N} \sum_{\mathbf{p}}^{\Lambda} \frac{1}{p^{k}}$ was used in (17) which is a truncated formula in the following sense:

1. The nonanalytic term proportional to $\tau^{d / 2}$, see the last but one loop integral in (16), was neglected, as it is subleading in the region of space dimensions studied here, i.e. $d>8$.

2. Higher than quartic $\phi$ terms in (17) were left out for the same reason. 
3. We restrict ourself to the model where quartic couplings are the highest order, i.e. neglecting the invariants in (3) which are there represented by the dots. A fifth order coupling, for instance, would provide a contribution $\sim I_{2} \times\left(\phi^{3}\right)^{\alpha \alpha}$ in Eq. (17).

\section{IV. $n$-DEPENDENT FREE ENERGY IN SPECIAL CASES}

Eq. (17) is now used to compute $\beta \Delta \Phi \equiv-\left\{\frac{1}{n N} \ln \overline{Z^{n}}-\frac{1}{n N}\left[\ln \overline{Z^{n}}\right]\right.$ para $\}$, i.e. the shift of the $n$ dependent free energy from the continuation of the paramagnetic one, in some special cases.

\section{A. Neglecting loops: the tree approximation}

Inserting the expansions from (B5) into (17), and neglecting loop terms, the mean field free energy of the model with nonzero $\tau, w, u_{1}, u_{2}, u_{3}, u_{4}$, and assuming infinite step RSB takes the form:

$$
\beta \Delta \Phi^{\mathrm{mf}}=\frac{1}{6} w^{-2} \tau^{3}+\left(\frac{1}{8} u_{1}+\frac{1}{24} u_{2}-\frac{1}{24} u_{3}\right) w^{-4} \tau^{4}+O\left(\tau^{5}\right)-\frac{1}{24} u_{4} w^{-4} n \tau^{4}-\frac{9}{640} u_{2}^{-3} w^{4} n^{5} .
$$

Inserting $w=1, u_{1}=3, u_{2}=2, u_{3}=-6$, and $u_{4}=0$, this gives the free energy of the SK model

$$
\beta \Phi^{\mathrm{SK}}=-\left[\ln 2+\frac{J^{2}}{4(k T)^{2}}\right]+\frac{1}{6} \tau^{3}+\frac{17}{24} \tau^{4}+O\left(\tau^{5}\right)-\frac{9}{5120} n^{5}, \quad 2 \tau=1-\left(\frac{k T}{J}\right)^{2} .
$$

This formula agrees with the results of Ref. [3] up to the order studied here. ${ }^{4}$ From Eq. (18) follows that infinite step RSB is only a necessary condition for the anomalous (non-Gaussian) free energy fluctuation: a nonzero $u_{4}$ produces a term linear in $n$. This may be a generic feature: interactions which are disconnected in replica space (like $\sum_{\alpha \beta \gamma \delta} \phi^{\alpha \beta^{2}} \phi^{\gamma \delta^{2}}$ ) generate Gaussian free energy fluctuations even at the level of the tree approximation.

\section{B. One-loop correction for the infinite step RSB case}

When substituting $u_{3}$ by $\bar{u}_{3} \equiv u_{3}+n u_{4}$, the $n$-dependent free energy shift takes the following simple form in the first order perturbative calculation [see Eqs. (17), (B5), (18)]:

$$
\beta \Delta \Phi=\beta \Delta \Phi^{(0)}+n \beta \Delta \Phi^{(1)}+\beta \Delta \Phi^{\text {anom }},
$$

\footnotetext{
${ }^{4}$ Unfortunately the definition of $\tau$ differs from that of Refs. [2, 3, 4], which is called here $\tau^{\prime}$. The simple relation between them is $\tau=\tau^{\prime}-\frac{1}{2} \tau^{\prime 2}$.
} 
with

$$
\begin{aligned}
\beta \Delta \Phi^{(0)} & =\frac{1}{6} w^{-2} \tau^{3}\left[1+\left(u_{1}+3 u_{2}-\bar{u}_{3}-4 u_{4}\right) I_{4}-8 w^{2} I_{6}\right]+\frac{1}{24} w^{-4} \tau^{4}\left[\left(3 u_{1}+u_{2}-\bar{u}_{3}\right)\right. \\
& +\frac{1}{3}\left(33 u_{1}^{2}+38 u_{1} u_{2}-26 u_{1} \bar{u}_{3}-24 u_{1} u_{4}+21 u_{2}^{2}-14 u_{2} \bar{u}_{3}-8 u_{2} u_{4}+5 \bar{u}_{3}^{2}+8 \bar{u}_{3} u_{4}\right) I_{4} \\
& \left.+8\left(5 u_{1}-u_{2}-\bar{u}_{3}+4 u_{4}\right) w^{2} I_{6}+24 w^{4} I_{8}\right], \\
\beta \Delta \Phi^{(1)} & =\frac{1}{6} w^{-2} \tau^{3}\left[\bar{u}_{3} I_{4}+4 w^{2} I_{6}\right]+\frac{1}{24} w^{-4} \tau^{4}\left[\frac { 1 } { 3 } \left(-9 u_{1}^{2}-4 u_{1} u_{2}+12 u_{1} \bar{u}_{3}+8 u_{1} u_{4}+4 u_{2} \bar{u}_{3}\right.\right. \\
& \left.\left.-8 u_{2} u_{4}-5 \bar{u}_{3}^{2}+8 u_{4}^{2}\right) I_{4}+4\left(u_{2}+4 u_{4}\right) w^{2} I_{6}+24 w^{4} I_{8}\right],
\end{aligned}
$$

and

$$
\begin{aligned}
\beta \Delta \Phi^{\text {anom }} & =-\frac{9}{640} u_{2}^{-4} w^{4}\left\{u_{2}+\left[-2 u_{1}^{2}+2(2 n-5) u_{1} u_{2}-9 u_{2}^{2}-8 u_{2} u_{3}-8 u_{2} u_{4}-2 u_{3}^{2}\right] I_{4}\right. \\
& \left.+4\left[-6 u_{1}+(n+7) u_{2}+6 \bar{u}_{3}-6 n u_{4}\right] w^{2} I_{6}-72 w^{4} I_{8}\right\} \times n^{5} .
\end{aligned}
$$

\section{For comparison: the replica symmetric free energy}

The RS mean field order parameter $q \equiv \phi^{\alpha \beta}$, for any $\alpha \neq \beta$, satisfies Eq. (B1) which now takes the form

$$
\tau=-\frac{1}{2}(n-2) w q-\frac{1}{6}\left[\left(n^{2}-3 n+3\right) u_{1}+u_{2}+(n-1) \bar{u}_{3}\right] q^{2} .
$$

This equation can be used to substitute $\tau$ for $q$ in the $\operatorname{RS}$ relations $\left(\phi^{2}\right)^{\alpha \alpha}=(n-1) q^{2}$, $\left(\phi^{3}\right)^{\alpha \alpha}=$ $(n-1)(n-2) q^{3},\left(\phi^{4}\right)^{\alpha \alpha}=(n-1)\left(n^{2}-3 n+3\right) q^{4}, \sum_{\beta} \phi^{\alpha \beta^{4}}=(n-1) q^{4}$. It is now straightforward to derive from Eq. (17) the free energy shift of the replica symmetric system with respect to the paramagnet:

$$
\beta \Delta \Phi_{\mathrm{RS}}=\beta \Delta \Phi_{\mathrm{RS}}^{(0)}+n \beta \Delta \Phi_{\mathrm{RS}}^{(1)}+n^{2} \beta \Delta \Phi_{\mathrm{RS}}^{(2)}+\ldots
$$

We can make the following observations:

- The RS free energy is a regular power series in $n$, with no anomalous part, and the terms $\beta \Delta \Phi_{\mathrm{RS}}^{(k)}$ all have the same character as a power series of $\tau$ starting with $\tau^{3}$.

- $\beta \Delta \Phi_{\mathrm{RS}}^{(0)}=\beta \Delta \Phi^{(0)}$ of Eq. (19) up to $\tau^{4}$ (inclusively).

- The leading term proportional to $\tau^{3}$ of $\beta \Delta \Phi_{\mathrm{RS}}^{(1)}$ is identical with the corresponding RSB contribution 
in Eq. (201). We have

$$
\begin{aligned}
\beta \Delta \Phi_{\mathrm{RS}}^{(1)} & =\frac{1}{6} w^{-2} \tau^{3}\left[\bar{u}_{3} I_{4}+4 w^{2} I_{6}\right]+\frac{1}{24} w^{-4} \tau^{4}\left[u_{2}+\frac{1}{3}\left(-7 u_{1}^{2}+10 u_{1} u_{2}+12 u_{1} \bar{u}_{3}+8 u_{1} u_{4}\right.\right. \\
& \left.\left.+21 u_{2}^{2}+8 u_{2} \bar{u}_{3}-16 u_{2} u_{4}-3 \bar{u}_{3}^{2}+8 u_{4}^{2}\right) I_{4}+2\left(4 u_{1}-8 u_{2}-4 \bar{u}_{3}+8 u_{4}\right) w^{2} I_{6}+48 w^{4} I_{8}\right] .
\end{aligned}
$$

- From the previous observations follows that the RS and RSB free energies differ only at the $O(5)$ order in the double series of $\tau$ and $n$.

The $O\left(n^{2}\right)$ term — which is missing in the RSB scheme at one-loop level — is given by

$$
\beta \Delta \Phi_{\mathrm{RS}}^{(2)}=-\frac{1}{24} w^{-2} \tau^{3}\left[1+\left(u_{1}+3 u_{2}-\bar{u}_{3}-4 u_{4}\right) I_{4}-8 w^{2} I_{6}\right]+O\left(w^{-4} \tau^{4}\right) .
$$

\section{THE NONANALYTIC TEMPERATURE DEPENDENCE OF THE FREE ENERGY}

As we have seen in the previous section, the RSB free energy starts to differ from the RS one only in the fifth analytic order $O\left(\tau^{5}\right), O\left(\tau^{4} n\right), O\left(\tau^{3} n^{2}\right)$, and — lastly but most importantly — in the anomalous

term $O\left(n^{5}\right)$. In dimensions not much higher than 8 , however, these terms are subdominate with respect to the nonanalytic one proportional to $\tau^{d / 2}$. For retrieving this nonanalytic contribution from the Gaussian free energy in Eq. (15), it is converted into the following equivalent form [Eq. (16) is not very useful for that purpose]:

$$
\ln Z_{G}=-\frac{1}{2} \sum_{j} \ln \frac{\Lambda^{2}+\bar{\lambda}_{j}}{\pi} \sum_{\mathbf{p}} 1+\frac{1}{d} \sum_{j} \sum_{\mathbf{p}} \frac{p^{2}}{p^{2}+\bar{\lambda}_{j}},
$$

and the last term gives the nonanalytic contribution $\ln Z_{G}^{\text {na }}$ which can be generally written as

$$
\frac{1}{n N} \ln Z_{G}^{\mathrm{na}}=\frac{1}{d} \frac{1}{N} \sum_{\mathbf{p}} p^{2} \frac{1}{n} \sum_{(\alpha \beta)} \bar{G}_{\alpha \beta, \alpha \beta} .
$$

\section{A. $\ln Z_{G}^{\text {na }}$ for the infinite step RSB sheme}

At first sight, computing the right hand side of Eq. (23) seems to be a formidable task due to the complicated structure of the Gaussian propagators; see Ref. [15] for details. Nevertheless, as we are interested in the leading nonanalytic temperature dependence, some important simplifications follow:

- This leading term comes from the near infrared region $p^{2} \sim \tau$; the propagators there were all listed in Sec. 6 of Ref. [15]. When computing this near infrared propagators, the quartic couplings can be neglected in the masses in Eq. (9), they enter only through the order parameter $\phi^{\alpha \beta}$. 
- The $n=0$ propagators — which were computed in [15] - may be used, as their $n$-dependence enters only at much higher orders. [This is just alike the order parameter function $q(x)$.]

- Beside the replica summations in Eq. 23, the origin of the relevant $n$-dependence is $x_{0}$; see (B4).

The trace in (23) can be written as an integral over the continuous overlap parameter $x$ : $\frac{1}{n} \sum_{(\alpha \beta)} \bar{G}_{\alpha \beta, \alpha \beta}=-\frac{1}{2} \int_{n}^{1} \bar{G}_{11}^{x x} d x$ and, by using Eq. (60) from Ref. [15], we can write

$$
\int_{n}^{1} p^{2} \bar{G}_{11}^{x x} d x=\left(1+2 t+2 t^{2}\right)(1-n)-2 \frac{1+8 t+8 t^{2}}{(1+2 t)^{2}} w^{2} \frac{1}{p^{4}} \int_{n}^{1} q(x)^{2} d x+\frac{8}{(1+2 t)^{2}} w^{4} \frac{1}{p^{8}} \int_{n}^{1} q(x)^{4} d x
$$

where the dimensionless variable $t \equiv w q_{1} / p^{2}$ was introduced. Eq. (B5) can be used to compute $\int_{n}^{1} q(x)^{2} d x=-\sum_{\beta} \phi^{\alpha \beta^{2}}=-\left(\phi^{2}\right)^{\alpha \alpha}$, and $\int_{n}^{1} q(x)^{4} d x=-\sum_{\beta} \phi^{\alpha \beta^{4}}$. Keeping only the relevant contributions, ${ }^{5}$ we arrive at

$$
\int_{n}^{1} p^{2} \bar{G}_{11}^{x x} d x=1+2 t-8 t^{3}+32 \frac{t^{4}(1+t)}{(1+2 t)^{2}}-\left(1+2 t+2 t^{2}\right) n+\frac{81}{10} \frac{1}{p^{4}\left(p^{2}+2 w q_{1}\right)^{2}} w^{8} u_{2}^{-4} n^{5} .
$$

We are now in the position to detach the leading nonanalytic temperature dependence in Eq. (23); approximating $w q_{1} \approx \tau$,

$$
\begin{gathered}
\text { for } d>8 \text { infinite step RSB : } \\
\frac{1}{n N} \ln Z_{G}^{\text {na }}=-\frac{16}{d} \int^{\infty} \frac{d^{d} p}{(2 \pi)^{d}} \frac{p^{2}+1}{p^{6}\left(p^{2}+2\right)^{2}} \times \tau^{d / 2}-\frac{81}{20 d} \int^{\infty} \frac{d^{d} p}{(2 \pi)^{d}} \frac{1}{p^{4}\left(p^{2}+2\right)^{2}} \times w^{8} u_{2}^{-4} \tau^{d / 2-4} n^{5} .
\end{gathered}
$$

[Dimensional regularization is to be understood here, allowing the ultraviolet cutoff $\Lambda$ go to infinity. This same remark is applicable for the RS case, Eq. (27).] Note the lack of the $\tau^{d / 2} n$ term in the RSB scheme. The $O\left(\tau^{d / 2-4} n^{5}\right)$ contribution is negligible; see Eq. (21).

\section{B. $\ln Z_{G}^{\text {na }}$ for the RS case}

Eq. (23) is now simplified as

$$
\frac{1}{n N} \ln Z_{G}^{\mathrm{na}}=\frac{n-1}{2 d} \frac{1}{N} \sum_{\mathbf{p}} p^{2} \bar{G}_{1}
$$

with the diagonal propagator $\bar{G}_{1}$ satisfying [16]

$$
\frac{1}{2} n(n-1) \bar{G}_{1}=\frac{1}{p^{2}+\bar{\lambda}_{L}}+(n-1) \frac{1}{p^{2}+\bar{\lambda}_{A}}+\frac{1}{2} n(n-3) \frac{1}{p^{2}+\bar{\lambda}_{R}}
$$

\footnotetext{
${ }^{5}$ Terms with an extra $x_{1}$ factor are subleading, and thus unimportant here. Their calculation using the near infrared propagators from Ref. [15] would even be inconsistent, since corrections to these propagators give the same (subleading) order.
} 
where the three masses are in leading order

$$
\bar{\lambda}_{L}=2 \tau, \quad \bar{\lambda}_{A}=-\frac{4}{n-2} \tau, \quad \text { and } \quad \bar{\lambda}_{R}=-n \frac{2}{n-2} \tau .
$$

Extracting the terms providing nonanalytic temperature dependence, Eq. (25) takes the following form:

$$
\begin{gathered}
\text { for } d>8 \quad \mathrm{RS}: \\
\frac{1}{n N} \ln Z_{G}^{\mathrm{na}}=-\frac{16}{d} \int^{\infty} \frac{d^{d} p}{(2 \pi)^{d}} \frac{p^{2}+1}{p^{6}\left(p^{2}+2\right)^{2}} \times \tau^{d / 2}-\frac{8}{d} \int^{\infty} \frac{d^{d} p}{(2 \pi)^{d}} \frac{p^{2}+1}{p^{4}\left(p^{2}+2\right)^{3}} \times \tau^{d / 2} n .
\end{gathered}
$$

A comparison of Eqs. (24) and (27) shows that the leading nonanalytic free energy contributions of the RSB and RS phases coincide, and the free energy difference is $O(5)$ in the double series of $\tau$ and $n$ down to dimensions 8. In the next section, the line in the $\tau, n$ plane where the free energy difference disappeares is computed in first order perturbation theory taking into account the $O(5)$ analytical terms.

\section{THE LINE OF EQUAL FREE ENERGIES OF THE RSB AND RS SCHEMES FOR $d>8$}

To find the fifth order (in the double series of $\tau$ and $n$ ) results, we must do two further steps:

- The one-replica quantities in Eq. (17) must be extended to the appropriate order by using the formulae in appendix B (for RSB) and in subsection IV C (for RS).

- Eq. (17) must be supplemented by the fifth order terms.

As for the first step, the notation $\delta \ldots$ is introduced to mean the difference of a one-replica quantity in the two schemes; $\delta\left(\phi^{2}\right)^{\alpha \alpha} \equiv\left(\phi^{2}\right)_{\mathrm{RSB}}^{\alpha \alpha}-\left(\phi^{2}\right)_{\mathrm{RS}}^{\alpha \alpha}$ for instance. Then we have

$$
\begin{aligned}
\delta\left(\phi^{2}\right)^{\alpha \alpha} & =-\frac{1}{9} w^{-6} u_{2}^{2} \tau^{4}+\frac{1}{3} w^{-4} u_{2} n \tau^{3}-\frac{1}{4} w^{-2} n^{2} \tau^{2}+O(5), \\
\delta\left(\phi^{3}\right)^{\alpha \alpha} & =\frac{2}{5} w^{-7} u_{2}^{2} \tau^{5}-w^{-5} u_{2} n \tau^{4}+\frac{1}{2} w^{-3} n^{2} \tau^{3}+\frac{27}{80} w^{3} u_{2}^{-3} n^{5}+O(6), \\
\delta \sum_{\beta} \phi^{\alpha \beta^{4}} & =-\frac{8}{15} w^{-6} u_{2} \tau^{5}+w^{-4} n \tau^{4}-\frac{81}{80} w^{4} u_{2}^{-4} n^{5}+O(6) ;
\end{aligned}
$$

whereas $\delta\left(\phi^{4}\right)^{\alpha \alpha}$ and $\left(\left(\phi^{2}\right)^{\alpha \alpha}\right)^{2}$ are of order $O(6)$.

Inclusion of the three fifth order one-replica quantities $\left(\phi^{5}\right)^{\alpha \alpha}, \sum_{\beta} \phi^{\alpha \beta^{3}}\left(\phi^{2}\right)^{\alpha \beta}$, and $\left(\phi^{2}\right)^{\alpha \alpha}\left(\phi^{3}\right)^{\alpha \alpha}$ into Eq. (17) is not an easy task. Fortunately, however, the $\delta$ 's formed of them are at most of order $O(6)$, and thus negligible for the present purpose. 
We can now search for the line in the $\tau$-n plane where the RS and infinite step RSB free energies coincide. Inserting $n=c w^{-2} u_{2} \tau$ into Eq. (28), the following — somewhat surprising — result turns up:

$$
\begin{aligned}
\delta\left(\phi^{2}\right)^{\alpha \alpha} & =-\frac{1}{4}(c-2 / 3)^{2} w^{-6} u_{2}^{2} \tau^{4}, \\
\delta\left(\phi^{3}\right)^{\alpha \alpha} & =\frac{9}{80}(c-2 / 3)^{2}\left(3 c^{3}+4 c^{2}+4 c+8\right) w^{-7} u_{2}^{2} \tau^{5}, \\
\delta \sum_{\beta} \phi^{\alpha \beta^{4}} & =-\frac{3}{80}(c-2 / 3)^{2}\left(27 c^{3}+36 c^{2}+36 c+32\right) w^{-6} u_{2} \tau^{5} .
\end{aligned}
$$

We can write the free energy difference between the RSB and RS phases up to one-loop order, using Eqs. (11) and (17), in terms of $c$ as follows:

$$
\beta\left(\Phi^{\mathrm{RSB}}-\Phi^{\mathrm{RS}}\right) \cong-\frac{1}{16}\left(c-\frac{2}{3}\right)^{2}\left[c-\frac{2}{3}-2 u_{2}^{-1} f_{d}(\Lambda)\right] w^{-6} u_{2}^{2} \tau^{5}, \quad c \rightarrow \frac{2}{3}
$$

where the correction term is

$$
f_{d}(\Lambda)=\frac{1}{3}\left(2 u_{1}^{2}+11 u_{1} u_{2}+12 u_{2}^{2}+7 u_{2} u_{3}+4 u_{2} u_{4}+2 u_{3}^{2}\right) I_{4}+4\left(2 u_{1}-3 u_{2}-2 u_{3}\right) w^{2} I_{6}+24 w^{4} I_{8} .
$$

It can be seen from Eq. (29) that for the cases $c \approx 2 / 3$, the RSB and RS free energies differ only at 3-loop order! Without any further assumption, we should go up to that order to gain the 1-loop correction to $c_{\text {eq }}$, i.e. to the $c$ value of the line where the two free energies are equal. We will, however, assume that for $d>8$ the mean field form of Eq. (29), i.e.

$$
\beta\left(\Phi^{\mathrm{RSB}}-\Phi^{\mathrm{RS}}\right) \sim\left(c-c_{\mathrm{eq}}\right)^{3},
$$

remains valid for the model. Eq. (29) then provides $c_{\mathrm{eq}}=\frac{2}{3}\left[1+u_{2}^{-1} f_{d}(\Lambda)\right]$, and finally

$$
\text { for } d>8 \quad \Phi^{\mathrm{RSB}}=\Phi^{\mathrm{RS}} \Longrightarrow n=\frac{2}{3}\left[u_{2}+f_{d}(\Lambda)+O(2 \text {-loop })\right] w^{-2} \tau \text {. }
$$

\section{BETWEEN SIX AND EIGHT DIMENSIONS}

\section{A. The infinite step RSB case}

As it has been explained in Ref. [15], the quartic vertex whose bare value is $u_{2}{ }^{6}$ suffers a change in its temperature scaling from $\tau^{0}$ to $\tau^{d / 2-4}$ when crossing $d=8$. The RSB order parameter is severely influenced by this, as best seen from Eqs. (10), (11) by extracting a term proportional to $q(x)^{3}$ from the one-loop contribution, see [15], and matching it to the corresponding zero-loop one:

$$
\frac{1}{3}\left[u_{2}+24 \int^{\infty} \frac{d^{d} p}{(2 \pi)^{d}} \frac{1}{p^{4}\left(p^{2}+2\right)^{2}} w^{4} \tau^{d / 2-4}\right] \times \phi^{\alpha \beta^{3}} .
$$

\footnotetext{
$\overline{{ }^{6} \text { In [15] }} u=u_{2} / 2$ was used.
} 
The second part between the brackets is called $\tilde{u}_{2}$ and reproduced in Appendix $\mathbb{C}$, it obviously dominates the bare value $u_{2}$. Beside $\tilde{u}_{2}$, the combination $\tilde{u}_{1}-\frac{1}{3} \tilde{\bar{u}}_{3}$ emerges too from the one-loop correction, as shown in Appendix [C, and the equation of state in (10) and (11), with the relevant terms kept only, takes the following form [the usual infinite step ultrametric ansatz is assumed here, and therefore $q(x)$ replaces $\left.\phi^{\alpha \beta}\right]:$

$$
2 \tau q(x)+w\left[\left(n-\frac{2}{3} x_{0}\right) q_{0}^{2}-2\left(q_{1}-\frac{1}{2} x_{1} q_{1}\right) q(x)-\frac{1}{3} x q(x)^{2}\right]+\left(\tilde{u}_{1}-\frac{1}{3} \tilde{\bar{u}}_{3}\right) q_{1}^{2} q(x)+\frac{1}{3} \tilde{u}_{2} q(x)^{3}=0 .
$$

Solving this equation provides the order parameter function in this transitional domain of dimensions:

$$
q(x)=\frac{w}{\tilde{u}_{2}} x, \quad \tau=w q_{1}-\frac{1}{2}\left(\tilde{u}_{1}+\tilde{u}_{2}-\frac{1}{3} \tilde{\tilde{u}}_{3}\right) q_{1}^{2}, \quad n=\frac{2}{3} x_{0}, \quad 6<d<8 ;
$$

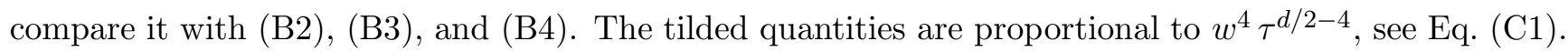
Note that although $x_{1} \cong \frac{\tilde{u}_{2}}{w^{2}} \tau \sim w^{2} \tau^{d / 2-3}$ is one-loop order, the order parameter $q(x)$ continues to be zero-loop order.

We are now proceeding to compute the relevant nonanalytic terms to the free energy in (14): both $\mathcal{L}^{(0)}$ and $\ln Z_{G}$ must be considered in this regime of dimensions.

- Gaussian free energy: near infrared contribution. Eq. (24) remains valid, except replacing $u_{2}$ by $\tilde{u}_{2}$ in the second term, and exploiting the definition of $\tilde{u}_{2}$ in (C1):

$$
\frac{1}{n N} \ln Z_{G}^{\mathrm{na}}=-\frac{16}{d} \int^{\infty} \frac{d^{d} p}{(2 \pi)^{d}} \frac{p^{2}+1}{p^{6}\left(p^{2}+2\right)^{2}} \times \tau^{d / 2}-\frac{27}{160 d} w^{4} \tilde{u}_{2}^{-3} n^{5} .
$$

- A detailed analysis based on Ref. [15] shows that only highly subdominant contributions $\frac{1}{n N} \ln Z_{G}^{\text {na }} \sim \tau^{d-1}$ - result from the far infrared regime $p^{2} \sim u_{2} q^{2} \sim \frac{u_{2}}{w^{2}} \tau^{2}$; these are even smaller than the correction term from the near infrared regime which is $\sim \tau^{d / 2+1}$.

- Nonanalytic temperature dependence occurs in $\mathcal{L}^{(0)}$ of Eq. (4) due to the tilded quartic couplings in the order parameter, see (34). For retrieving the relevant leading contributions, it is sufficient to take

$$
-\frac{1}{n N} \mathcal{L}^{(0)}=\frac{1}{2} \tau\left(\phi^{2}\right)^{\alpha \alpha}+\frac{1}{6} w\left(\phi^{3}\right)^{\alpha \alpha},
$$

thus neglecting terms which are smaller by $u_{2} / \tilde{u}_{2} \sim \tau^{4-d / 2}$. A straightforward calculation provides

$$
-\frac{1}{n N} \mathcal{L}^{(0)}=C_{2 \text {-loop }} \times w^{2} \tau^{d-3}+\frac{9}{160} w^{4} \tilde{u}_{2}^{-3} n^{5} .
$$

(Note the lack of the term $\sim \tau^{d / 2}$.) The first term, which obviously dominates the subleading one $\sim \tau^{d / 2+1}$, consists of contributions like $w^{-1} \tilde{u}_{2}^{2} q_{1}^{5}$. A consistent calculation of $C_{2 \text {-loop }}$, however, requires the 2-loop extension of the equation of state. Similarly, 2-loop corrections behind the Gaussian free energy in Eq. (14) yield the same kind of term. 


\section{B. The replica symmetric case}

Similarly to the RSB case, we must include the one-loop term in Eq. (10), and - keeping only the relevant terms - the RS equation of state reads:

$$
2 \tau q+(n-2) w q^{2}+(n-2) w \frac{1}{N} \sum_{\mathbf{p}} \bar{G}_{2}=0,
$$

with [16]

$$
n(n-1)(n-2) \bar{G}_{2}=2(n-2) \frac{1}{p^{2}+\bar{\lambda}_{L}}+(n-1)(n-4) \frac{1}{p^{2}+\bar{\lambda}_{A}}-n(n-3) \frac{1}{p^{2}+\bar{\lambda}_{R}},
$$

and for the RS masses see (26). Extracting the nonanalytic contribution from the third term is somewhat lengthy but straightforward; in the zero replica number limit it can be put into the form [see Eq. (C1)]

$$
(n-2) w \frac{1}{N} \sum_{\mathbf{p}} \bar{G}_{2}=\left[\left(\tilde{u}_{1}-\frac{1}{3} \tilde{\bar{u}}_{3}\right)+\frac{1}{3} \tilde{u}_{2}\right] q^{3}, \quad n=0 .
$$

Note that the resultant RS equation of state is equivalent with the RSB one of Eq. (33) for $x=x_{0}=x_{1}$.

We are now in the position to analyse the two possible sources for the RS free energy having nonanalytic temperature dependence for $6<d<8$ :

- As for the Gaussian part, Eq. (27) remains valid for $\frac{1}{n N} \ln Z_{G}^{\text {na }}$ even in this dimensional regime.

- Using the RS equation of state from above, it is straightforward to see that the leading nonanalytic term to $-\frac{1}{n N} \mathcal{L}^{(0)}$ is proportional to $\tau^{d-3}$. It has, however, a 2-loop character, and a consistent calculation would require extending to the next perturbative order.

\section{The free energy difference between the RSB ans RS phases}

Collecting pieces of information from previous sections and subsections, namely Eqs. (22), (27), (35) and (36), the leading terms which do not cancel in the free energy difference when $6<d<8$ are the following:

$$
\begin{aligned}
& \beta\left(\Phi^{\mathrm{RSB}}-\Phi^{\mathrm{RS}}\right)= \\
& C_{2 \text {-loop }} \times w^{2} \tau^{d-3}+\frac{8}{d} \int^{\infty} \frac{d^{d} p}{(2 \pi)^{d}} \frac{p^{2}+1}{p^{4}\left(p^{2}+2\right)^{3}} \times \tau^{d / 2} n+\frac{1}{24} w^{-2} \tau^{3} n^{2}+\frac{9}{160 d}(3-d) w^{4} \tilde{u}_{2}^{-3} n^{5} .
\end{aligned}
$$

[The notation $C_{2 \text {-loop }}$ was kept here for simplicity, although it may obviously differ from that defined in Eq. (36). For the definition of $\tilde{u}_{2}$ see (C1).] The line where the RSB and RS free energies coincide follows from this equation:

$$
n \sim w^{2} \tau^{d / 2-3}, \quad 6<d<8
$$


The proportionality constant is a one-loop integral, whose value can only be computed from the knowledge of the two-loop integral $C_{2 \text {-loop }}$.

\section{THE STABILITY BOUNDARY OF THE RS PHASE}

It is well known from the famous finding of de Almeida and Thouless [10] that the mean field Ising spin glass in a homogeneous magnetic field enters the RSB phase along the boundary where the RS phase becomes unstable. This was later extended by Kondor [4] to the case when the magnetic field is zero, the replica number $n$, however, is finite: $n$ essentially takes over the role of the magnetic field, and along an Almeida-Thouless (AT) line in the $\tau-n$ plane, the RS phase becomes unstable. In [4] a simplified model — the "truncated" model with all the quartic couplings but $u_{2}$ zero — was used, and it was shown that the RS and RSB free energies coincide along the instability line.

In Ref. [12] the leading behaviour of the dangerous "replicon" mass $\Gamma_{\mathrm{R}}$ close to $T_{c}$ was expressed in terms of the exact cubic and quartic vertices $w^{\text {exact }}$ and $u_{2}^{\text {exact }}$, and also the exact order parameter $q^{\text {exact }}$ $\mathrm{as}^{7}$

$$
\Gamma_{\mathrm{R}}=n w^{\text {exact }} q^{\text {exact }}-\frac{2}{3} u_{2}^{\text {exact }} q^{\text {exact } 2}, \quad d>8 .
$$

The zero replicon mode signals the instability of the RS phase providing for the AT-line:

$$
n_{\mathrm{AT}}=\frac{2}{3} w^{\text {exact }-1} u_{2}^{\text {exact }} q^{\text {exact }}
$$

Substituting the bare values for the vertices and the mean field order parameter $w q=\tau$, agreement with the zero-loop result for the line of equal free energies of Eq. (32) is found, thus reproducing Kondor's result. (Although the more generic model with all the quartic couplings is considered here.)

Computing the one-loop correction of the replicon mass by standard perturbative methods is straightforward for $d>8$, although somewhat lengthy. Omitting the details, only the final result is displayed here:

$$
\begin{aligned}
& \Gamma_{\mathrm{R}}=\left\{1+\left[(n-1) u_{1}+u_{3}+4 u_{4}\right] I_{4}+(n-2) w^{2} I_{6}\right\} n\left(w q^{\text {exact }}\right)+\left\{\frac{1}{3}\left[n(n-3) u_{1}-2 u_{2}\right]\right. \\
+ & \frac{1}{9}\left[\left(2 n^{3}-5 n^{2}-3 n-4\right) u_{1}^{2}-12 u_{1} u_{2}+4 n(n-3) u_{1} u_{3}+24 n(n-3) u_{1} u_{4}-18 u_{2}^{2}-24 u_{2} u_{3}-48 u_{2} u_{4}-4 u_{3}^{2}\right] I_{4} \\
+ & \left.\frac{1}{3}\left[-\left(2 n^{3}-6 n^{2}+12 n+16\right) u_{1}+24 u_{2}-2\left(n^{2}-8\right) u_{3}+8 n(n-6) u_{4}\right] w^{2} I_{6}-\left(2 n^{3}-9 n^{2}+12 n+16\right) w^{4} I_{8}\right\} q^{\text {exact }} 2 .
\end{aligned}
$$

\footnotetext{
7 The superscript "exact" is used to distinguish these quantities from the "bare" ones, which are the zeroth order contributions to them.
} 
This must be complemented by the equation of state, i.e. by the one-loop relationship between the order parameter and $\tau$ :

$$
\begin{array}{r}
-2 \tau=(n-2)\left\{1+\frac{1}{3}\left[(n-2) u_{1}-3 u_{2}-(n-2) u_{3}-\left(n^{2}-n-8\right) u_{4}\right] I_{4}-(n-2) w^{2} I_{6}\right\} \\
\left(w q^{\text {exact }}\right) \\
+O\left(q^{\text {exact } 2}\right) .
\end{array}
$$

From these two equations, we can easily conclude the leading behaviour for $n \sim \tau \ll 1$ :

$$
\begin{aligned}
\Gamma_{\mathrm{R}}=\left[1+\frac{1}{3}\left(-u_{1}+3 u_{2}+\right.\right. & \left.\left.u_{3}+4 u_{4}\right) I_{4}-4 w^{2} I_{6}\right] n \tau-\frac{2}{3}\left[u_{2}+\frac{1}{3}\left(2 u_{1}^{2}+10 u_{1} u_{2}+15 u_{2}^{2}\right.\right. \\
& \left.\left.+8 u_{2} u_{3}+8 u_{2} u_{4}+2 u_{3}^{2}\right) I_{4}+8\left(u_{1}-2 u_{2}-u_{3}\right) w^{2} I_{6}+24 w^{4} I_{8}\right] w^{-2} \tau^{2} .
\end{aligned}
$$

The instability line is obtained from the condition $\Gamma_{\mathrm{R}}=0$ :

$$
n_{\mathrm{AT}}=\frac{2}{3}\left[u_{2}+f_{d}(\Lambda)\right] w^{-2} \tau
$$

where the correction term has been defined in (30). Comparing this expression with (32), we can conclude that the mean field type behaviour persists for $d>8$ : the RS phase becomes unstable where its free energy coincides with that of the RSB phase. (See, however, the discussion in the Conclusion part.)

\section{CONCLUSION}

Two basic features of the mean field Ising spin glass were followed in this paper, by perturbatively taking into account the effect of the geometry of a high dimensional lattice ( $d$ certainly larger than 6 ). These properties are:

- the anomalous sample to sample free energy fluctuations (considering only large deviations),

- and the equality of the free energies of the replica symmetric and infinite step replica symmetry broken phases along the line (Almeida-Thouless line) where the RS phase becomes unstable.

Both problems can be elaborated by studying the $n$-dependent free energy below the spin glass transition.

As for the first item, perturbations break down the anomalous behaviour, and Gaussian large deviations take over the lead. As it was shown in Sec. IV, Gaussian fluctuation is common for any ansatz of the order parameter with the property of replica eqivalence (not to be confused with replica symmetry), i.e. it must be a geometrical effect. As it was pointed out by G. Parisi [17], Gaussian fluctuations always dominate whenever local interactions are inhomogeneous: this is certainly the case in the finite 
dimensional geometry of a hypercubic lattice (but not for the SK model). The fact that locally inhomogeneous interactions imply Gaussian large deviations of the free energy has been demonstrated recently on the Bethe lattice with finite connectivity, and continuously distributed quenched interactions [18]. The anomalous $n$-dependence of the RSB free energy, however, does persist, although it is subleading in finite dimensions.

The coincidence of the RS instability line and the line of equal free energies with the RSB phase is somewhat misterious even in the SK model: the free energy difference is of fifth order (in the double series in $\tau$ and $n$ ), as contrasted with the replicon mass wich is a second order quantity. We found that this feature of the mean field theory persists for $d>8$, but in a rather nontrivial way (see the complicated correction term in Eq. (30)). We must emphasize that the two computations in Secs. VI and VIII are completely independent. This result gives an important support to the scenario, at least for $d>8$, that replica symmetry must be broken by the infinite ultrametric hierarchy of the mean field spin glass proposed by Parisi. We must remember, however, that the condition of Eq. (31) was a priori assumed, and any conclusions depend on the validity of it.

In the dimensional domain $6<d<8$ the situation is more complicated. The AT line can be computed relatively easily, as it was in Ref. [13]:

$$
n_{\mathrm{AT}}=\frac{2}{3} w^{-2} \tilde{u}_{2} \tau \sim w^{2} \tau^{d / 2-3}
$$

see (C1) and Eq. (47) of [13]. Among the leading contributions to the free energy difference, which are now nonanalytic, there are terms which can be computed only by extending the calculation to two-loop order; as explained in Sec. VII This seems to be unfeasible, and no a priori assumptions are available now. A real miracle would be the coincidence of the two lines, presumably due to several cancellations. One can speculate that otherwise the separation of the two lines might be explained by some non mean field scenarios: first order transition or replica symmetry breaking with replica equivalence, but not with the infinite step ultrametric structure.

\section{Acknowledgments}

This work has benefited from a useful correspondence with Giorgio Parisi and Tommaso Rizzo. Sending me preliminary results of Ref. [18] is also acknowledged. 


\section{APPENDIX A: TRACES OF POWERS OF THE GAUSSIAN MASS OPERATOR FOR A GENERIC RSB SCHEME WITH REPLICA EQUIVALENCE}

Traces appearing in the formula for $\ln Z_{G}$, Eq. (16), can be computed and arranged into the generic form

$$
\frac{1}{n} \sum_{j} \bar{\lambda}_{j}^{k}=\frac{1}{n} \operatorname{Tr} \bar{M}^{k} \sim \tau^{k}+\left[\text { expression of }\left(\phi^{2}\right)^{\alpha \alpha}\left(\phi^{3}\right)^{\alpha \alpha}\left(\phi^{4}\right)_{,}^{\alpha \alpha} \sum_{\beta} \phi^{\alpha \beta^{4}}, \ldots\right] .
$$

These formulae are derived by two subsequent steps:

- Substitution of the mass components from (9).

- From the zero-loop equation of state $\bar{H}_{\alpha \beta}=0$, with $m=-\tau$ in (11), and exploiting replica equivalence new, replica independent equations follow, like this:

$$
\tau\left(\phi^{2}\right)^{\alpha \alpha}=-\frac{1}{2} w\left(\phi^{3}\right)^{\alpha \alpha}-\frac{1}{6}\left[u_{1}\left(\phi^{4}\right)^{\alpha \alpha}+u_{2} \sum_{\beta} \phi^{\alpha \beta^{4}}+\left(u_{3}+n u_{4}\right)\left(\left(\phi^{2}\right)^{\alpha \alpha}\right)^{2}\right] .
$$

In this way, we can get rid of terms where $\tau$ and $\phi$ are coupled.

The following formulae are valid for any RSB scheme compatible with replica equivalence, not necessarily ultrametric:

$$
\begin{aligned}
& \frac{1}{n} \operatorname{Tr} \bar{M}=-(n-1) \tau-\frac{1}{6}\left[(2 n-1) u_{1}+3 u_{2}+(n+1) u_{3}+\left(n^{2}-n+4\right) u_{4}\right]\left(\phi^{2}\right)^{\alpha \alpha} ; \\
& \frac{1}{n} \operatorname{Tr} \bar{M}^{2}=2(n-1) \tau^{2}+(n-2) w^{2}\left(\phi^{2}\right)^{\alpha \alpha}-\frac{1}{3} w\left[u_{1}+3 u_{2}+(n-1) u_{3}+\left(n^{2}-n-4\right) u_{4}\right]\left(\phi^{3}\right)^{\alpha \alpha} \\
& -\frac{1}{18} u_{1}\left[(2 n-3) u_{1}+6 u_{2}+2(n-1) u_{3}+2\left(n^{2}-n-8\right) u_{4}\right]\left(\phi^{4}\right)^{\alpha \alpha}+\frac{1}{18}\left[2 u_{1}^{2}-4(n-2) u_{1} u_{2}+3 u_{2}^{2}\right. \\
& \left.-2(n-5) u_{2} u_{3}-2\left(n^{2}-n-8\right) u_{2} u_{4}+2 u_{3}^{2}\right] \sum_{\beta} \phi^{\alpha \beta^{4}}+\frac{1}{18}\left[(3 n-4) u_{1}^{2}+12 u_{1} u_{2}+8 u_{1} u_{3}-(n-3) u_{3}^{2}\right. \\
& \left.-2\left(n^{2}-n-8\right) u_{3} u_{4}-n\left(n^{2}-n-8\right) u_{4}^{2}\right]\left(\left(\phi^{2}\right)^{\alpha \alpha}\right)^{2} \\
& \frac{1}{n} \operatorname{Tr} \bar{M}^{3}=-4(n-1) \tau^{3}+2(n-2) w^{3}\left(\phi^{3}\right)^{\alpha \alpha}+\frac{1}{2} w^{2}\left[(2 n-7) u_{1}-3 u_{2}-(n-1) u_{3}-\left(n^{2}-n+4\right) u_{4}\right]\left(\phi^{4}\right)^{\alpha \alpha} \\
& +w^{2}\left[-2 u_{1}+(n+1) u_{2}+2 u_{3}\right] \sum_{\beta} \phi^{\alpha \beta^{4}}+\frac{1}{2} w^{2}\left[-(6 n-19) u_{1}-3 u_{2}+(n-5) u_{3}+\right. \\
& \left.\left(n^{2}-n+4\right) u_{4}\right]\left(\left(\phi^{2}\right)^{\alpha \alpha}\right)^{2}+\ldots \\
& \frac{1}{n} \operatorname{Tr} \bar{M}^{4}=8(n-1) \tau^{4}+w^{4}\left[(3 n-4)\left(\phi^{4}\right)^{\alpha \alpha}+8 \sum_{\beta} \phi^{\alpha \beta^{4}}+2(n-7)\left(\left(\phi^{2}\right)^{\alpha \alpha}\right)^{2}\right]+\ldots
\end{aligned}
$$




\section{APPENDIX B: ZERO-LOOP ORDER PARAMETER OF THE $\tau, u_{1}, u_{2}, u_{3}, u_{4}$ MODEL}

For the model with higher than quartic invariants neglected — which is somewhat more general than the traditional "truncated" model where $u_{1}=u_{3}=u_{4}=0-$, the mean field equation of state follows from Eqs. (10) and (11) by neglecting the 1-loop term and setting $m=-\tau$ :

$$
\tau \phi^{\alpha \beta}=-\frac{1}{2} w\left(\phi^{2}\right)^{\alpha \beta}-\frac{1}{6}\left[u_{1}\left(\phi^{3}\right)^{\alpha \beta}+u_{2} \phi^{\alpha \beta^{3}}+\left(u_{3}+n u_{4}\right)\left(\phi^{2}\right)^{\alpha \alpha} \phi^{\alpha \beta}\right] .
$$

(Replica equivalence was applied to get the last term.) The infinite-step ultrametric solution of this equation can be represented by the order parameter function $q(x)$, see [9] and references therein, and $x$ falls into the interval $[n, 1]$ for $n>0$ small but finite. An application of the ultrametric replica algebra [19], and extending the method used for the truncated model [20], a somewhat lengthy calculation leads to

$$
q(x)= \begin{cases}q_{0} & n \leq x \leq x_{0} \\ K \frac{x}{\sqrt{1+\frac{u_{1}}{u_{2}} x^{2}}} & x_{0} \leq x \leq x_{1} \\ q_{1} & x_{1} \leq x \leq 1\end{cases}
$$

and the constant $K$ is best expressed by $x_{1}$ as

$$
K=\frac{w}{u_{2}} \frac{\sqrt{1+\frac{u_{1}}{u_{2}} x_{1}^{2}}}{1+\frac{u_{1}}{u_{2}} x_{1}} .
$$

Continuity of $q(x)$ at $x_{0}$ and $x_{1}$ means that only two of the four parameters $x_{0}, x_{1}, q_{0}$, and $q_{1}$ are independent. Choosing $x_{0}$ and $q_{1}$ as such, the most economic way to give them as function of the model parameters is the inverse relationship:

$$
\begin{aligned}
n & =\frac{2}{3} x_{0}+O(3), \\
\tau & =w q_{1}-\frac{1}{2}\left[u_{1}+u_{2}-\frac{1}{3}\left(u_{3}+n u_{4}\right)\right] q_{1}^{2}-\frac{1}{9} \frac{u_{2} u_{3}}{w} q_{1}^{3}+O(4) .
\end{aligned}
$$

[Orders $O(\ldots)$ are understood in the sense of the double series in $x_{0}$ and $q_{1}$. Notice the lack of $O(2)$ term in the first equation.]

The invariants entering Eq. (17) can be computed by the methods presented in [19], and they have 
the following expansions:

$$
\begin{aligned}
&\left(\phi^{2}\right)^{\alpha \alpha}=-q_{1}^{2}+\frac{2}{3} x_{1} q_{1}^{2}+O(5)=-w^{-2}\left[\tau^{2}+\frac{1}{3} w^{-2}\left(3 u_{1}+u_{2}-u_{3}-n u_{4}\right) \tau^{3}+\ldots\right], \\
&\left(\phi^{3}\right)^{\alpha \alpha}= 2 q_{1}^{3}-2 x_{1} q_{1}^{3}+\frac{2}{5} x_{1}^{2} q_{1}^{3}+\frac{2}{45} x_{0}^{2} q_{0}^{3}+O(6)=w^{-3}\left[2 \tau^{3}+w^{-2}\left(3 u_{1}+u_{2}-u_{3}-n u_{4}\right) \tau^{4}+\ldots\right] \\
&+\frac{27}{80} w^{3} u_{2}^{-3} n^{5}, \\
&\left(\phi^{4}\right)^{\alpha \alpha}=-3 q_{1}^{4}+4 x_{1} q_{1}^{4}+O(6)=-3 w^{-4} \tau^{4}+\ldots, \\
& \sum_{\beta} \phi^{\alpha \beta^{4}}=-q_{1}^{4}+\frac{4}{5} x_{1} q_{1}^{4}-\frac{2}{15} x_{0} q_{0}^{4}+O(6)=-w^{-4} \tau^{4}+\ldots-\frac{81}{80} w^{4} u_{2}^{-4} n^{5}, \\
&\left(\left(\phi^{2}\right)^{\alpha \alpha}\right)^{2}=q_{1}^{4}-\frac{4}{3} x_{1} q_{1}^{4}+O(6)=w^{-4} \tau^{4}+\ldots
\end{aligned}
$$

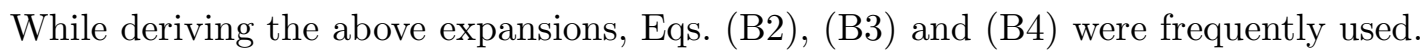

\section{APPENDIX C: CALCULATION OF THE NONANALYTIC CONTRIBUTIONS FOR THE EQUATION OF STATE}

Extracting the leading nonanalytic contributions from the second term in Eq. (10) becomes possible by the following simplifications:

- All the bare cubic vertices but $\bar{W}_{\alpha \beta, \beta \gamma, \gamma \alpha}=w$ are negligible, see Eq. (12).

- We can use the bare propagators in the near infrared region [15]; this is equivalent by neglecting the bare quartic couplings $u_{1}, u_{2}, u_{3}$, and $u_{4}$ in the mass components in (9).

- The single replica sum arising, see below, is then approximated by evaluating it in the smallest ultrametric block of size $x_{1}-1$, and $x_{1}$ is obviously negligible here for small $\tau$ and $d>6$.

We can write then

$$
\frac{1}{2} \sum_{(\gamma \delta),(\mu \nu)} \bar{W}_{\alpha \beta, \gamma \delta, \mu \nu} \bar{G}_{\gamma \delta, \mu \nu} \cong w \sum_{\gamma \neq \alpha \beta} \bar{G}_{\alpha \gamma, \beta \gamma} \cong-2 w \bar{G}_{1 x}^{x x_{1}},
$$

$x$ being the ultrametric distance between $\alpha$ and $\beta$, and the parametrization of ultrametric matrices was used in the last formula [15]. Eq. (62) of Ref. [15] with $y=x_{1}$ can now be used:

$$
p^{2} \bar{G}_{1 x}^{x x_{1}}=\frac{\left(1+6 t+4 t^{2}-4 t_{x}^{2}\right) t_{x}}{(1+2 t)^{2}}=t_{x}+2 t t_{x}-8 \frac{(1+t) t^{2} t_{x}}{(1+2 t)^{2}}-4 \frac{t_{x}^{3}}{(1+2 t)^{2}}
$$

where $t \equiv w q_{1} / p^{2}$ and $t_{x} \equiv w q(x) / p^{2}$. Only the last two terms contribute to the nonanalytic temperature dependence we are looking for: the second term in Eq. (10) can then be written as

$$
\left(\tilde{u}_{1}-\frac{1}{3} \tilde{\bar{u}}_{3}\right) q_{1}^{2} q(x)+\frac{1}{3} \tilde{u}_{2} q(x)^{3}
$$


with

$$
\begin{aligned}
\tilde{u}_{1}-\frac{1}{3} \tilde{\bar{u}}_{3} & =16 \int^{\infty} \frac{d^{d} p}{(2 \pi)^{d}} \frac{p^{2}+1}{p^{6}\left(p^{2}+2\right)^{2}} w^{4} \tau^{d / 2-4}, \\
\tilde{u}_{2} & =24 \int^{\infty} \frac{d^{d} p}{(2 \pi)^{d}} \frac{1}{p^{4}\left(p^{2}+2\right)^{2}} w^{4} \tau^{d / 2-4} .
\end{aligned}
$$

[1] A. Crisanti, G. Paladin, H.-J. Sommers, and A. Vulpiani, J. Phys. I (France) 2, 1325 (1992).

[2] G. Parisi and T. Rizzo, Phys. Rev. Lett. 101, 117205 (2008).

[3] G. Parisi and T. Rizzo, Phys. Rev. B 79, 134205 (2009), arXiv:0811.1524.

[4] I. Kondor, J. Phys. A 16, L127 (1983).

[5] D. Sherrington and S. Kirkpatrick, Phys. Rev. Lett. 35, 1792 (1975).

[6] A. J. Bray and M. A. Moore, J. Phys. C 12, 79 (1979).

[7] T. Temesvári, C. De Dominicis, and I. R. Pimentel, Eur. Phys. J. B 25, 361 (2002), cond-mat/0202162.

[8] T. Aspelmeier and M. Moore, Phys. Rev. Lett. 90, 177201 (2003).

[9] M. Mézard, G. Parisi, and M. A. Virasoro, Spin Glass Theory and Beyond, vol. 9 of Lecture Notes in Physics (World Scientific, Singapore, 1987).

[10] J. R. L. de Almeida and D. J. Thouless, J. Phys. A 11, 983 (1978).

[11] T. Temesvári, Phys. Rev. B 78, 220401(R) (2008), arXiv:0809.1839.

[12] T. Temesvári, J. Phys. A 39, L61 (2006), cond-mat/0510209.

[13] T. Temesvári, Nucl. Phys. B 772, 340 (2007), arXiv:cond-mat/0612523.

[14] G. Parisi, Int. J. Mod. Phys. B 18, 733 (2004).

[15] C. De Dominicis, I. Kondor, and T. Temesvári, Beyond the Sherrington-Kirkpatrick Model (World Scientific, 1998), vol. 12 of Series on Directions in Condensed Matter Physics, p. 119, cond-mat/9705215.

[16] E. Pytte and J. Rudnick, Phys. Rev. B 19, 3603 (1979).

[17] G. Parisi, private communication.

[18] G. Parisi and T. Rizzo (2009), arXiv:0910.4553.

[19] M. Mézard and G. Parisi, J. Phys. I (France) 1, 809 (1991).

[20] G. Parisi, J. Phys. A 13, 1887 (1980). 